Trivent Publishing

(C) The Authors, 2015

Available online at http://trivent-publishing.eu/

Philosophy, Communication, Media sciences Series

Volume Saint Gerard of Cenad: Tradition and Innovation

\title{
Die Rolle des hl. Gerhard im sozio-kulturellen Leben der Banater Christen
}

\author{
Franz Metz \\ Gerhardskult und Gerhardslegende bei den Banater Schwaben \\ in Rumänien und Deutschland
}

\begin{abstract}
Nur kurze Zeit nach dem Märtyrertod von Bischof Gerhard begann man im Banat seiner in Legenden und Berichten zu gedenken. Im Laufe der Jahrhunderte hat dieser Gerhardskult viele Änderungen erlebt, ihm zu Ehren wurden Gedichte geschrieben und Lieder komponiert. Selbst nach der Teilung seines ehemaligen Csanader Bistums nach dem ersten Weltkrieg wurde er in allen drei Nachfolgediözesen nicht nur als erster Bischof des Banats sondern auch als ein Heiliger geehrt. Durch die Folgen des zweiten Weltkriegs, die Vertreibung der Donauschwaben aus den südslawischen Ländern unddie spätere Auswanderung des größten Teils der Banater Schwaben nach Deutschland gelangte der Gerhardskult auch in dieses Land. In Stuttgart wurde so das St. Gerhards-Werk gegründet, in München aber das Gerhardsforum Banater Schwaben.
\end{abstract}

Keywords: Banat; Hl. Gerhard; Mittelalter; Donauschwaben; Kommunismus; Rumänien; Deutschland; Gerhardsforum; Gerhardswerk

This is an Open Access article distributed in accordance with the Creative Commons Attribution Non Commercial (CC-BYNC-ND 4.0) license, which permits others to copy or share the article, provided original work is properly cited and that this is not done for commercial purposes. Users may not remix, transform, or build upon the material and may not distribute the modified material (http://creativecommons.org/licenses/by-nc/4.0/)

DOI: 10.22618/TP.PCMS.20151.176010 
Durch die aus den Ländern Ex-Jugoslawiens und Ungarns 1944-45 vertriebenen Donauschwaben und durch die aus dem rumänischen Banat nach dem Zweiten Weltkrieg ausgewanderten Katholiken wurde der Gerhardskult auch nach Deutschland verpflanzt. Dadurch konnte in der Zeit des Eisernen Vorhangs wenigstens durch die in Deutschland lebenden Donauschwaben aus dem Südosten Europas die Erinnerung an den hl. Gerhard wachgehalten werden. Man suchte nach dem Beispiel anderer deutscher Hilfswerke (z.B. Bonifatiuswerk) einen Namen, um seine Identität und seine Herkunft am besten zu verdeutlichen. Der Name von Bischof Gerhard war deshalb für die aus den südöstlichen deutschen Siedlungsgebieten angekommenen katholischen Flüchtlingen, Vertriebenen und Aussiedlern geeignet, um ihre Identität am besten zu verdeutlichen. Für viele aus dem Banat stammenden Aussiedler bedeutete das Stuttgarter Gerhardswerk auch eine Hilfe in der Bewältigung ihrer ersten Schritte in der Bundesrepublik Deutschland. So manche Hilfen für Kirchen und Seelsorger des Temeswarer Bistums kamen zwischen 1960-1989 auch von diesem Hilfswerk.

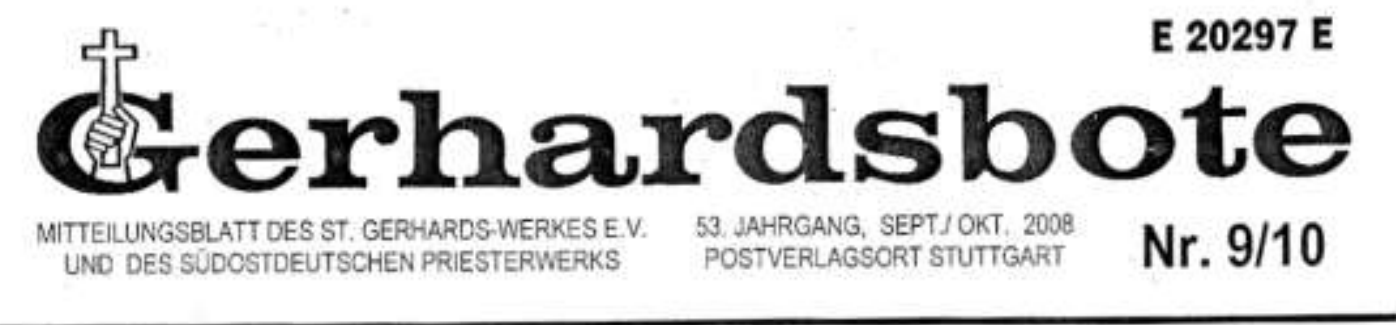

Bild. 1. Titelseite des Mitteilungsblattes „Gerhardsbote,“ Stuttgart (Ausschnitt)

Das St. Gerhards-Werk ist aus dem „Arbeitskreis südostdeutscher Katholiken e.V.“, gegründet 1952 in München, hervorgegangen. Im Jahre 1955 wurde dieser Arbeitskreis in St. Gerhards-Werk e.V. umbenannt und im Jahre 1957 fand dessen Übersiedlung nach Stuttgart statt. Seit dann ist dieser kirchliche Verein in der Diözese Rottenburg-Stuttgart angesiedelt. Dazu wurde auch ein Parallelverein für jüngere Mitglieder ins Leben gerufen, die „Gerhardsjugend“. Diese war dem Verein besonders in der Organisation von Wallfahrten, Wochenendtagungen und Auslandsfahrten behilflich. Ab 1958 wurde das St. Gerhards-Werk vom Jesuitenpater Wendelin Gruber geleitet, der kurz davor durch Intervention von Bundeskanzler Dr. Konrad Adenauer aus jugoslawischer Haft freigekommen war. Er war auch der Begründer der seit 1959 jährlich stattfindenden großen Gelöbniswallfahrt der Donauschwaben nach Altötting (Bayern). Sein Nachfolger war ab 1965 Prälat Josef Haltmayer, der u.a. auch das Mitteilungsblatt Gerhardsbote regelmäßig herausgegeben hat. Dieses Mitteilungsblatt erscheint auch heute noch zweimonatlich mit einem Umfang von 12 Seiten.

Das St. Gerhards-Werk setzte sich seit dem Beginn seiner Existenz ein für die Integration der Vertriebenen und Aussiedlern in Deutschland, organisierte regelmäßig Studientage zur Geschichte und Religion der Donauschwaben, Eingliederungsseminare für Heimatvertriebene und Aussiedler, Wallfahrten, Maiandachten und Gottesdienste. Im Rahmen des St. Gerhards-Werks wurden auch einige Bücher und Sammelbände zur Kirchengeschichte der Donauschwaben veröffentlicht. Heute gehört das St. Gerhards-Werk zum Arbeitskreis Katholischer Vertriebenenorganisationen der Diözese Rottenburg-Stuttgart. 


\section{GERHARDSFORUM}

Mitteilungsblatt des Gerhardsforums Banater Schwaben e.V. Mai 2011 (Heft 4)

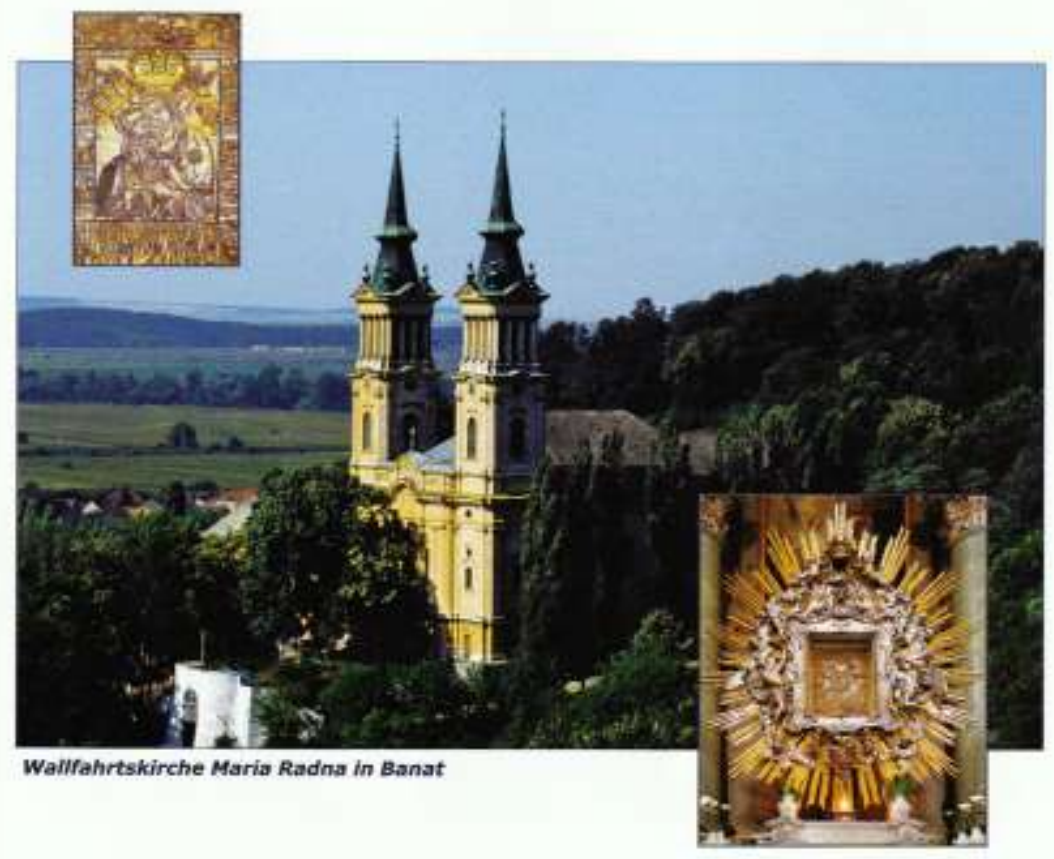

Maria Radna: Der Wallfahrtsort und die Basilika Maria Radna im Banat + Fin wur derthatiger Waldschatten + Die Banater Schwaben und ihre Kirchen + Wallfahrten + Gedanken uber das Pilgern + Informationsabend uber Maria Radna im Rumanischen Generalkonsulat München + Zukunft für Maria Radna + Partnerschaft zwischen Maria Ramersdorf in Bavern und Maria Radna im Banat + + + Glaube: Das Halleluja der Osternacht + Bischof Martin Roos wirdigt den Heiligen Gerhard von Sagredo + Temeswarer Domchor "Exultate” singt in Subotica + Pater Berno hat ein Herz fur die Rumänen + Mutig für das Wort Gottes in zwei Diktaturen eingetreten + Papst Benedikt XVI. - Apostolische Reise nach Deutschland +++ Kultur: Franz Liszt zum 200. Geburtstag + Franz Liszts Konzerte im Banat + Rudolf Hollinger - Zum 100. Geburtstag + + + Publikationen: Katholisches Gesangbuch der Donauschwaben + Erstes Buch 0ber Maria Radna in rumanischer Sprache + + +

Bild 2. Titelseite des Mitteilungsblattes „Gerhardsforum,“ München

Das Gerhardsforum Banater Schwaben e.V. wurde im Jahre 2009 in München ins Leben gerufen. Als Logo des Vereins hat man das in den Sarkophag aus Tschanad eingemeiselte Kreuz genommen. $\mathrm{Zu}$ den Zielen und Aufgaben dieses kirchlichen Vereins zählt u.a. die Pflege der kirchlichen Traditionen der Banater Schwaben und Donauschwaben, die Aufarbeitung und Bekanntmachung der Kirchengeschichte der Banater Diözesen, die Veranstaltung von Wallfahrten, Gottesdiensten, Maiandachten, Symposien und Kirchenkonzerten, die Veranstaltung von Studienfahrten und Gedenktagen wie auch die Unterstützung von verschiedenen kirchlichen Projekten im Banat. Der Verein gibt zweimal jährlich das umfangreiche Mitteilungsblatt GERHARDSFORUM heraus und setzt sich ein für den Ausbau Zusammenarbeit zwischen den landsmannschaftlichen Organisationen in Deutschland und den Kirchen im Banat. So wurde 2011 die Partnerschaft zwischen den beiden Wallfahrtskirchen Maria Ramersdorf in München und Maria Radna im Banat ins Leben gerufen. Diese wird stets lebendig gehalten durch gegenseitige Besuche, gemeinsamen Wallfahrten und durch einen regen Austausch. Am 31. August 2014 fand in St. Pius, München, gemeinsam mit Renovabis (Freising) ein Symposium statt, bei welchem die Situation der Heimatkirchen im Banat und über verschiedene kirchliche Hilfsprojekte gesprochen wurde. Auch die Ökumene spielt dabei eine große 
Rolle. Im Jahre 2011 wurde im Rahmen des Gerhardsforums das Katholische Gesangbuch der Donauschwaben veröffentlicht.

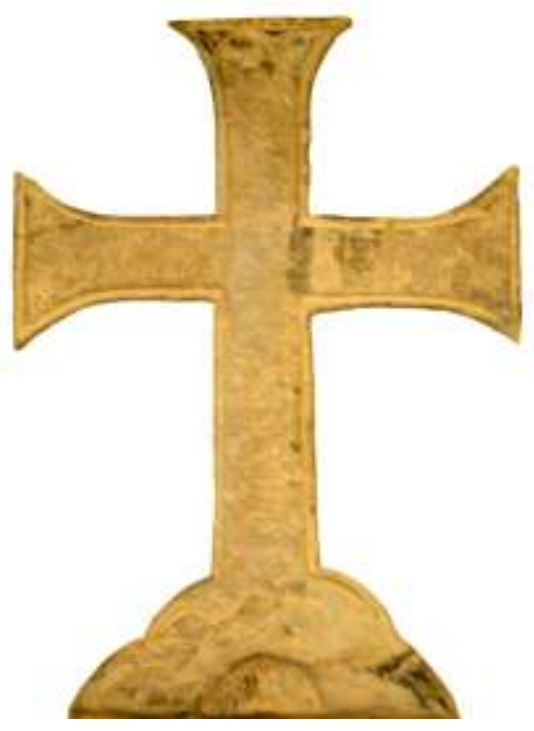

Bild 3. Logo des Gerhardsforums Banater Scheaben e.V., München: das Kreuz auf dem Sarkophag des hl. Gerhard in Tschanad

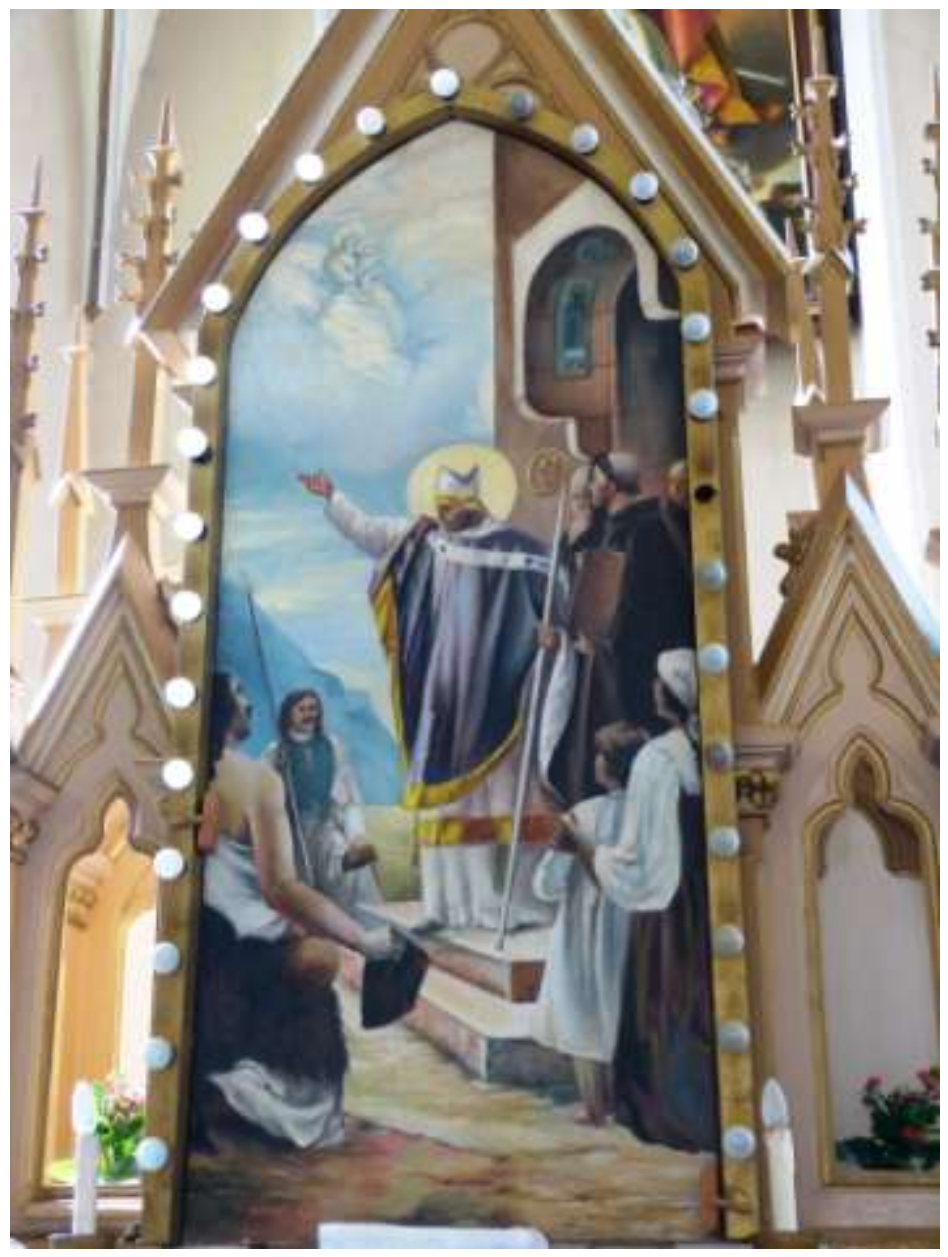

Bild 4. 10 Hauptaltarbild der römisch-katholischen Kirche in Schag (Josef Schweininger: Hl. Gerhard) 
In der Zwischenkriegszeit versuchte man durch zahlreiche Publikationen den Gerhardskultus im Banat auch in deutscher Sprache wieder zu verbreiten. Nach nur wenigen Jahren ist zum Beginn des 20. Jahrhunderts bereits die Erscheinung des Temeswarer St. Gerardusblattes eingestellt worden und auch das in Werschetz erschienene Mitteilungsblatt Sct. Gerardus. Religiöses Sonntagsblatt für das katholische Volk hatte keine lange Lebensdauer. So erschienen z.B. in deutschsprachigen Banater Zeitungen nach 1919 Artikeln von Béla Schiff, im Katholischen Volkskalender brachte man mehrmals verschiedene Aufsätze über das Leben und Wirken des hl. Gerhard und man begann von kirchlicher Seite aus die Pfarrkirche von Tschanad als Wallfahrtskirche zu etablieren. Pfarrer Dr. Koloman Juhász veröffentlichte in der Zwischenkriegszeit seine Arbeiten zur Kirchengeschichte des Mittelalters wie auch seine Forschungen zum Wirken des hl. Gerhard. Der erste Bischof des Banats wurde so in den kleinsten katholischen Pfarrgemeinden bekannt und im Religionsunterricht konnte die Schuljugend darüber Einzelheiten erfahren. So malte z.B. um 1930 der Kantorlehrer Josef Schweininger aus Schag ein Bild für den Hauptaltar seiner Heimatkirche - eigentlich eine Kopie nach dem bekannten Gerhardsbild von Gyula Túry aus der Zeit 1896/97.

Bischof Martin Roos hat in seiner Arbeit Gerhard von Csanád - Protomärtyrer des christlichen Ungarn aus dem Jahre 1991 über dessen Persönlichkeit und Verehrung ausführlich geschrieben.

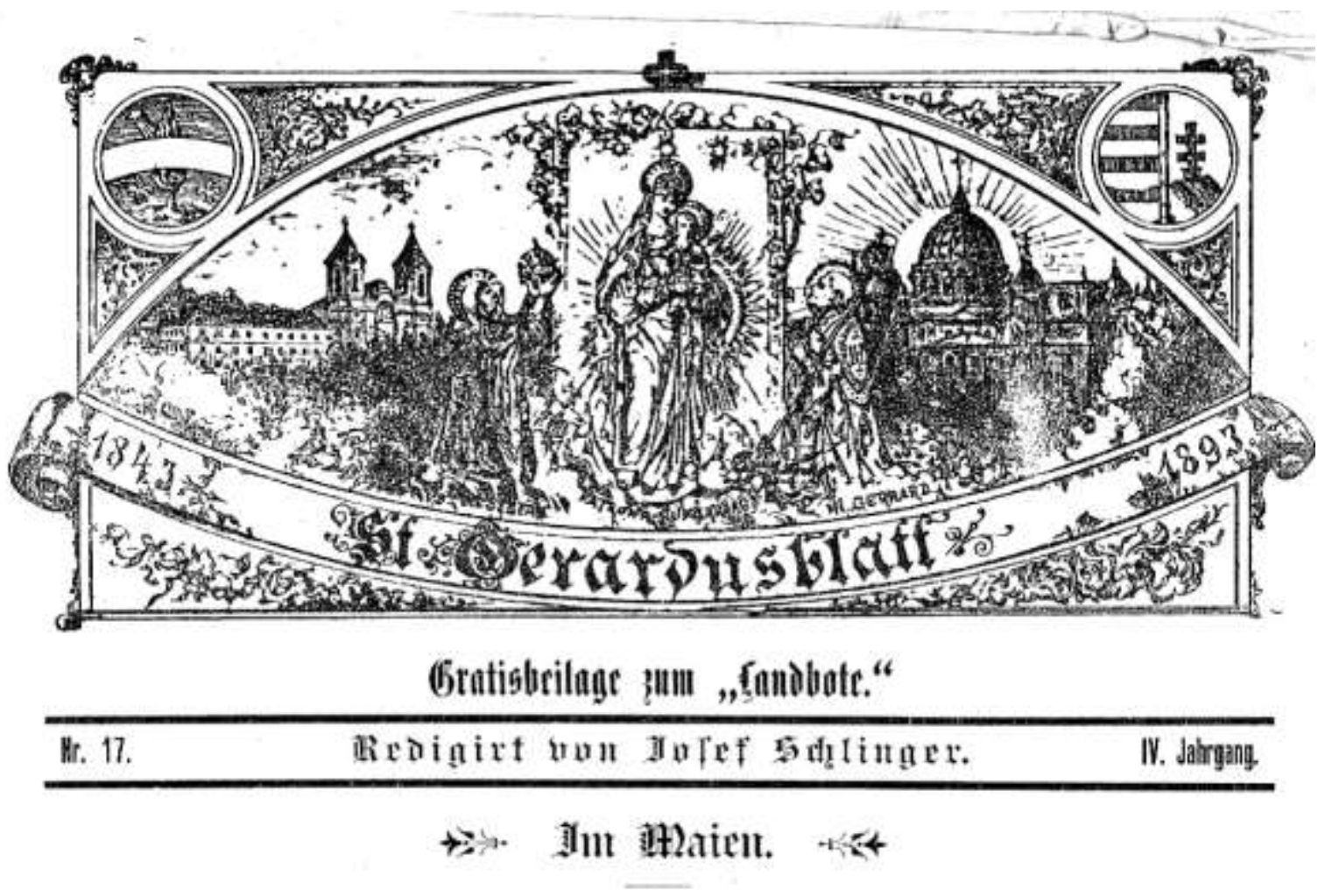

Bild 5. Titelseite der Schrift „Gerhardusblatt“ (Ausschnitt)

Die kirchliche Situation änderte sich im Banat schlagartig 1947 mit dem Verbot der konfessionellen Schulen in der Diözese Temeswar, den kirchlichen Restriktionen und der Einschränkung des religiösen Lebens. Ab Herbst 1944 wurden die Banater Schwaben aus dem Serbischen Banat vertrieben, ab Januar 1945 wurden viele Banater Schwaben aus dem rumänischen Banat zur Zwangsarbeit nach Russland deportiert, danach in den Baragan. Der Kreuzweg der katholischen Kirche des Banats und ganz Rumäniens hat damit begonnen. Bis zur Wende von 1989 war die römisch-katholische Kirche in Rumänien nur noch eine geduldete Kirche und viele Priester und Bischöfe wurden erst Anfang der 60er Jahre aus den kommunistischen Gefängnissen entlassen. Trotz der Unterdrückung durch die totalitäre kommunistische rumänische Staatsmacht, wurde in den Reihen der katholischen Gläubigen versucht, die bisherigen kirchlichen Traditionen aufrecht zu erhalten. Und dazu gehört auch die Verehrung des hl. Gerhard.

Gerade in der schwersten Zeit von Verfolgung, Not und Unterdrückung, im Jahre 1946, entstand das bekannteste Gerhardslied, das Messlied zu Ehren des hl. Gerhard, dessen Text von Pfarrer Dr. 
Franz Kräuter stammt und von den beiden Kantoren Hans Weisz und Paul Wittmann vertont wurde. Dieses mehrstrophige Lied entstand anlässlich des 900. Todestags des hl. Gerhard und wurde am 24. September 1946 zum ersten Mal gesungen. Im Sinne der traditionellen Messlieder war jede Strophe für einen bestimmten Teil des Ordinariums vorgesehen. Paul Wittmann hat die im ungarischen Volkston gehaltene Melodie von Hans Weisz für verschiedene Besetzungen harmonisiert.

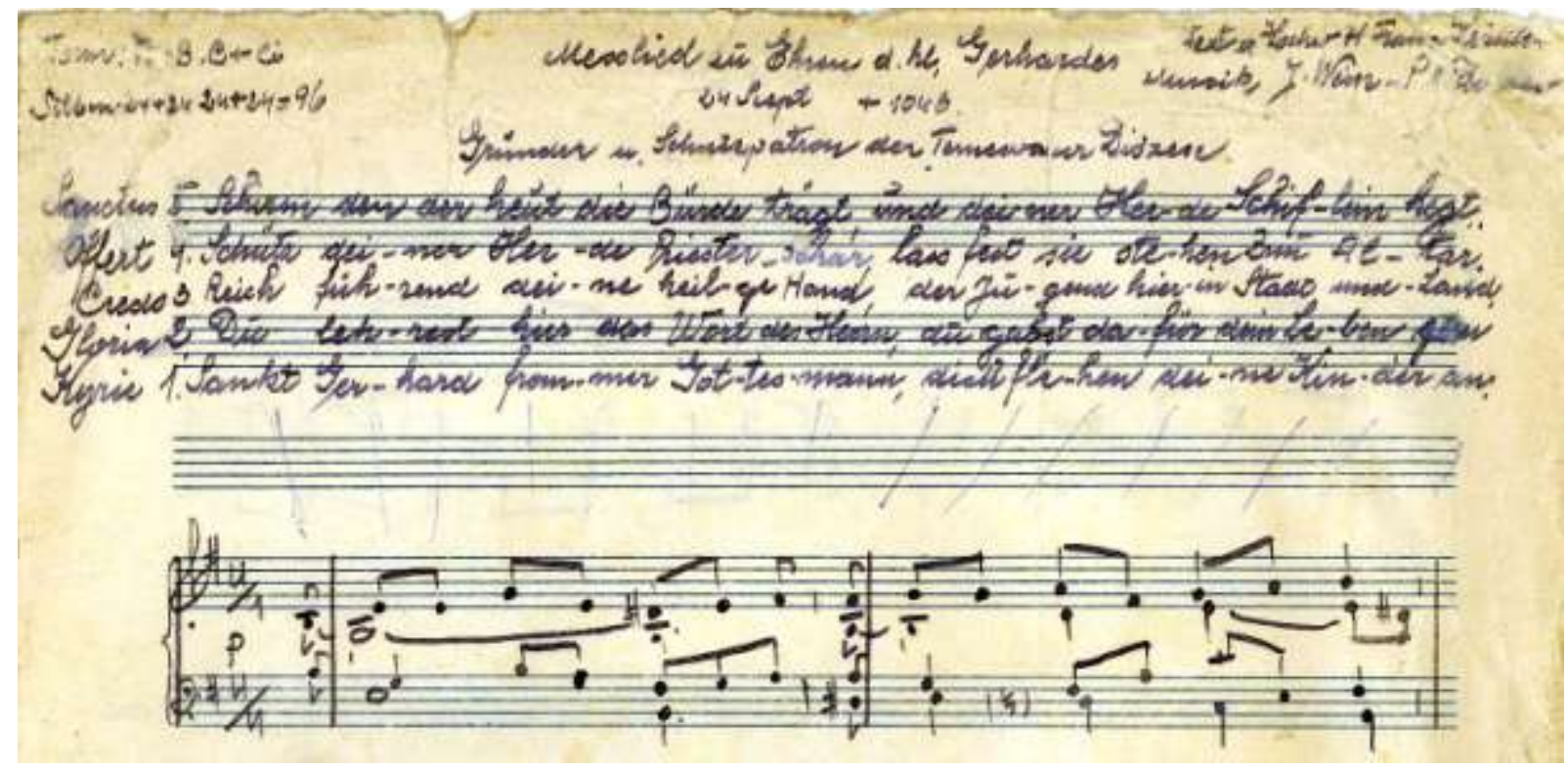

Bild 6. Manuskript des Gerhardsliedes (Temeswar 1946)

1. Sankt Gerhard, frommer Gottesmann, dich flehen deine Kinder an,

o Schutzpatron

bei Gottesthron!

$\mathrm{Zu}$ dir ruft unsres Lebens Not, sei unser Helfer jetzt bei Gott

in Angst und Not,

vor Krieg und Tod.

(Refr.)

Schau gnädig auf das Ackerfeld, das du als Sämann einst bestellt.

Hüt deine Saat,

schirm das Banat.

Du kamst als Gottes Bote her,

du pflanztest hier des Kreuzes Lehr.

Hüt deine Saat,

schirm das Banat!

2. Du lehrtest hier das Wort des Herrn, du gabst dafür dein Leben gern.

Du Gottes Held,

von ihm bestellt!

Mehr unsern Glauben, mach ihn stark,

lass treu uns sein und ohne Arg.

$\mathrm{O}$, steh uns bei,

hilf uns aufs neu. (Refr.)

3. Reich führend deine heilge Hand, 
der Jugend hier in Stadt und Land.

Führ sie zurück

zum wahren Glück.

Dass Glaube, Reinheit, Ehr und Treu

die Richtung ihres Lebens sei,

im Lebensstreit,

sei hilfbereit. (Refr.)

4. Schütz deiner Herde Priesterschar, lass fest sie stehen zum Altar.

Lass ihre Reihn

dem Herrn sich weihn,

dass sie wie du mit frommem Sinn

uns führen zu dem Heiland hin.

Ihr Lebenswerk,

den Glauben stärk! (Refr.)

5. Schirm den, der heut die Bürde trägt

und deiner Herde Schifflein hegt.

Den Hirten hüt,

der uns jetzt führt.

Erfleh ihm Weisheit und Geduld,

zum Opfer Kraft und Gotteshuld.

Halt von ihm fern

des Unglücks Stern. (Refr.)

Trotzdem man dieses Lied nicht veröffentlichen durfte, wurde es in fast allen katholischen Kirchengemeinden verbreitet und meist am Tag des hl. Gerhard, also am 24. September, im Gottesdienst gesungen.

Derselbe Text wurde etwas später von Msgr. Paul Lackner, der viele Jahre als Priester in Reschitza tätig war, vertont. Dieses Banater Gerhardslied ist in der Melodie (ebenfalls in Moll) einfacher und schlichter gehalten als die Vertonung von Weisz und Wittmann und war außerhalb Reschitzas kaum bekannt.

Die Tradition der Gerhardslieder vor1946 ist im Banat recht überschaubar. Wir kennen zwar die Titel einiger dieser Lieder, doch fehlt der Rest in den schriftlichen Überlieferungen. So wurde im Kantorenbuch der Kirchengemeinde Traunau zwar ein Lied zu Ehren des hl. Gerardus, als dem Patron der Csanáder Diöcese genannt, doch hat der Kopist das Lied nicht mehr aufgeschrieben.

Im Jahre 1980 kam der Auftrag vom damaligen Ordinarius, anlässlich des Gerhardjubiläums ein neues Gerhardslied zu komponieren, das aber zum Singen einfacher sein sollte als jenes aus dem Jahre 1946. Der Text stammt aus der Feder von Hans Mokka:

1. Gepriesen sei dein Name

und strahlend über Zeit,

vollbrachtest Wunderbares

in der Vergangenheit.

Geheiligt sei dein Name,

gepriesen deine Tat,

du warst der erste Bischof,

Sankt Gerhard im Banat.

2. Du richtest auf die Menschen

in ihrem Bittgebet,

gibst jedem innre Ruhe

der vor dir dankend steht.

Geheiligt sei dein Name, gepriesen deine Tat,

du warst der erste Bischof, 
Sankt Gerhard im Banat.

3. Du schützest uns vor Argwohn, steh bei in unsrer Not, du schützest vor der Armut, reichst jedem Heil und Brot. Geheiligt sei dein Name, gepriesen deine Tat, du warst der erste Bischof, Sankt Gerhard im Banat.

\section{DER GERHARDSWEG}

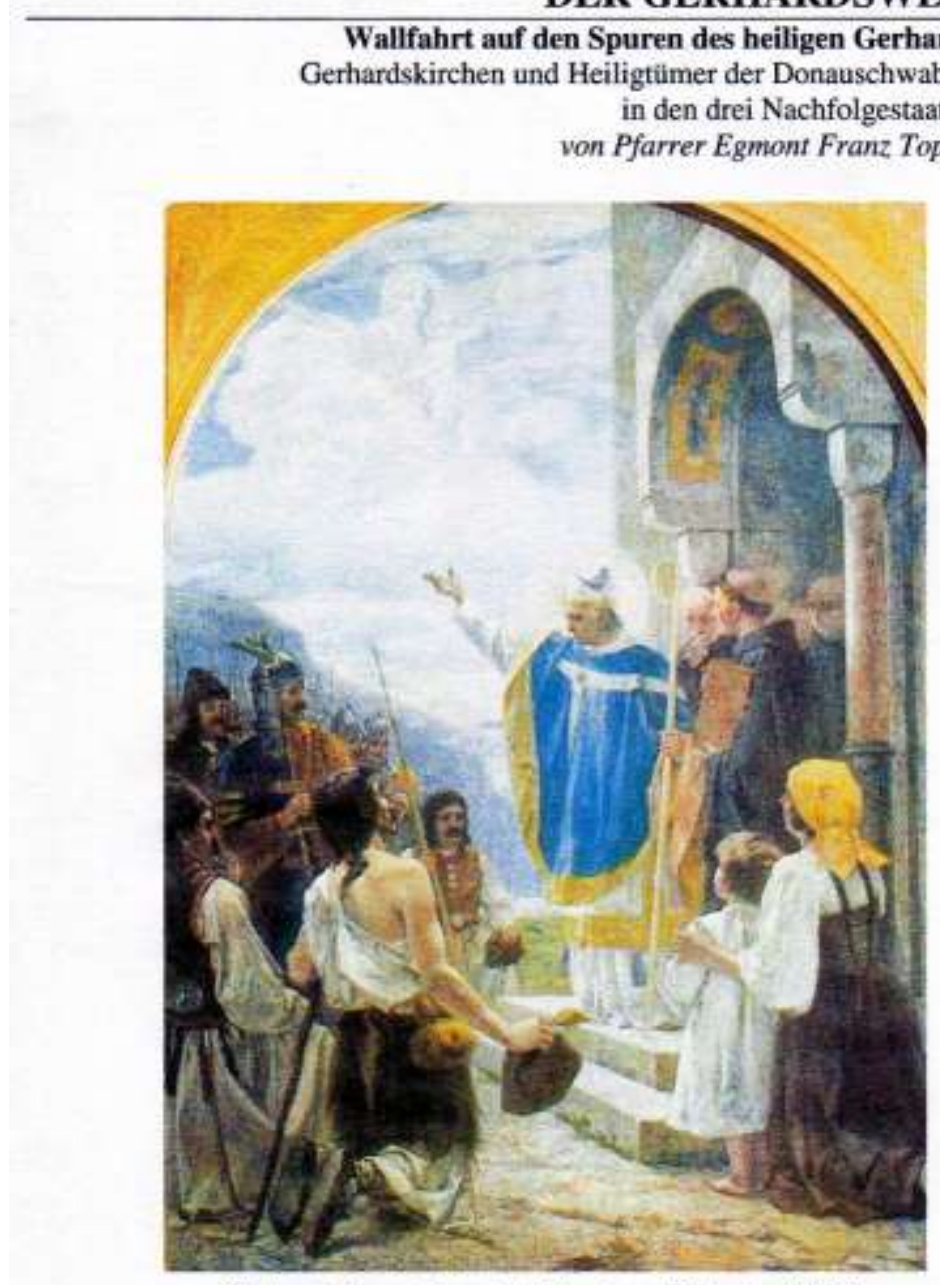

Gerhardsforum Banater Schwaben, München 2013

Bild 7. Titelseite der Broschüre „Der Gerhardsweg“ (verfasst von Pfarrer Egmont Franz Topits)

In einer kleineren Auflage hat das Gerhardsforum Banater Schwaben (München) anlässlich der Studienreise im Jahre 2013 ins Banat die wertvolle Arbeit von Pfarrer Egmont Franz Topits veröffentlicht: Der Gerhardsweg. Wallfahrt auf den Spuren des heiligen Gerhard. Gerhardskirchen und Heiligtümer der Donauschwaben in den drei Nachfolgestaaten. Es ist eine geschickt zusammengestellte Handreichung für jeden Wallfahrer, der sich europaweit auf den Weg macht, auf den Spuren des hl. Gerhard zu pilgern. Geschichtliche Daten, Gebete, Gesänge, Meditationen und viele touristisch Tipps lassen diese Broschüre kurzweilig erscheinen. Darin finden wir auch eine weniger bekannte Gerhardshymne von Emerich Csicsáky, das der ehemalige Pfarrer von Neuarad, 
Johann Baptist Mayer (1872-1935) aus dem Ungarischen übersetzt hat. Die Melodie stammt von F. Gaal.

1. Sankt Gerhard preiset unser Lied, Sankt Stefans treuem Freunde, der sein heimatliches Gefild verließ zu unserer Freude. Und im schönen Pannonien ein großer Apostel war, der des Heidenvolkes Schrecken, der Christen Schutz immerdar.

2. Im altehrwürdigen Tschanad der erste Bischof war er.

Drum segnete mit Fried' und Gnade Ihm tausendfach Gott der Herr. Oh, könnte ich Euch erzählen, was Gerhard für uns getan, dass das Kreuz zum Hort wir wählen und dies' Land Christi sei dann!

3. Segne deine Schäflein alle! Deine Treuen segne sie! Dass unsre Seel' nicht verfalle, vor dem Bösen rette sie.

Später Zeiten schwache Kinder Hilf nun finden deine Spur das Kreuz glänze herrlich wieder und die Tugend blühe nur.

4. Hohe, hehre Schutzfrau Ungarns mit Gerhard bete zu Gott!

Dein treues Volk auch bitte, warn's wenn öffnet sich der Hölle Pfort'. Für Christus hast einst gelitten St. Gerhard du treuer Hirt. Oh mögest du Gott nun bitten dass unsre Seel' nicht verirrt. 


\section{References}

Juhász, Koloman. Die Stifte der Tschanader Diözese im Mittelalter. Ein Beitrag zur Frühgeschichte und Kulturgeschichte des Banats. Münster in Westfalen, 1927.

. A Csanádi Püspökség Története. Alapitásától a Tatárjárásig (1030-1242). Makó 1930.

. Gerhard der Heilige, Bischof von Maroschburg, München: 1930.

Dániel, Géza und Ábrahám István. Szent Gellért és a csanádi püspökség rövid története. Szeged: 1980.

Dedek, Ludwig Crescens. Das Leben des Heiligen Märtyrers Gerardus, ersten Bischofs von Csanad. Budapest: 1900.

Roos, Martin. Erbe und Auftrag. Die alte Diözese Csanád. Band 1. Temeswar: 2009.

„Gerhard von Csanád - Protomärtyrer des christlichen Ungarn,“ in: Glaube in Volk und Heimat. Festgabe für Prälat Josef Haltmayer. Stuttgart: 1991.

Topits, Egmont Franz. Der Gerhardsweg. Wallfahrt auf den Spuren des heiligen Gerhard. Gerhardskirchen und Heiligtümer der Donauschwaben in den drei Nachfolgestaaten. München: 2013.

Archiv des St. Gerhards-Werkes, Diözese Rottenburg-Stuttgart.

Archiv des Gerhardsforums Banater Schwabene.V., München. 